\title{
ALAIN MABANCKOU ET L'ÉCRITURE DE LA MIGRANCE: ENJEUX ÉTHIQUES ET SOCIOPOÉTIQUES
}

\author{
Aetius BASSINTSA-BOUESSO
}

\author{
Université Gaston Berger de Saint-Louis, Sénégal
}

aetiusbirahima@yahoo.fr

Article reçu le 4 septembre 2017 | révisé depuis le 15 septembre 2017 | accepté le 15 décembre 2017

\begin{abstract}
RÉSUMÉ. La question des migrations relève d'une actualité brûlante. Les médias en font un traitement de nature à mettre en émoi le plus grand nombre et dans l'élan d'un patriotisme ou encore d'un ethnocentrisme exacerbé (selon les cas), des discours xénophobes se font de plus en plus entendre. La production romanesque d'Alain Mabanckou se réapproprie les questionnements que suscitent les mouvements migratoires qui concernent l'axe Afrique/ Europe, mais elle analyse également les mobilités sur les axes Afrique/ Afrique, village/ ville, etc. L'auteur s'érige ainsi en figure de proue de la littérature migratoire. La présente étude, en s'appuyant sur une lecture sociopoétique de ses textes, analyse les champs vers lesquels son écriture se déploie pour sonder les implications de ce phénomène afin de construire un discours littéraire le présentant comme un processus de repeuplement du monde actuel, qui pose aux espaces $\mathrm{d}$ 'intersection des peuples le défi de la construction de communautés homogènes et paisibles.
\end{abstract}

Mots-clés: Alain Mabanckou, diasporique, engagement, éthique, immigration, migrant.

\begin{abstract}
The migration is a major pressing issue. The media make it a treatement likely to stir up the largest number of poeple and the impetus of patriotism or an ethnocentrism exacerbated (according to the cases), xenophobic discourse is increasingly heard. Alain Mabanckou novelistic production reappropriates the questions raised by migratory movements concerning the Africa/ Europe axis, but also analyzes mobility on the Africa / Africa, Village / city axes, etc. The author thus stands out as a leading figure in migratory literature. This present study, conducted by the sociopoetic method, investigate the fields to which his writing deploys to probe the implications of this phenomenon in order to construct a literary discourse presenting it as a process of repopulation of the current world, which poses to the intersecting spaces of peoples the challenge of building homogeneous and peaceful communities.
\end{abstract}

Keywords: Alain Mabanckou, diasporic, commitment, ethics, immigration, migrant. 


\section{INTRODUCTION}

L'immigration est devenue une question majeure de la vie politique et sociale. Dans le domaine de la littérature, notamment celle que produit l'Afrique francophone, sa problématique bénéficie d'un regain d'intérêt et acquière une sorte de légitimité. Il en est ainsi aux yeux des écrivains, mais aussi pour le grand public. Dans son essai critique consacré à ce sujet, Albert (2005) analyse la manière dont le roman d'Afrique francophone, depuis l'époque coloniale jusqu'à nos jours, traite du thème des migrations selon une variété de configurations narratives. Or Mabanckou est ce qu'on appelle aujourd'hui un auteur de la " Migritude ». Ce concept, employé par la critique contemporaine, désigne en effet la catégorie de romanciers à laquelle il appartient, originaires du continent noir et installés en Occident, essentiellement en France, "mettant à profit l'expérience qu'ils ont de leur monde en dépassant la peinture du sentiment de nostalgie [qui animait autrefois] les écrivains de la Négritude » (Malonga, 2007, p. 117). Que nous révèle alors la scène symbolique que constituent ses romans sur les questions migratoires? Quel est en outre son positionnement éthique en la matière?

S'il est vrai que les liens historiques, linguistiques et culturels qui rattachent la France à nombre de pays d'Afrique ont concouru à l'affluence des ressortissants de cet espace vers l'Hexagone, l'imaginaire social du sujet africain dans son rapport à $\mathrm{l}^{\prime}$ " ailleurs »n'est pas pour autant cristallisé exclusivement sur l'axe Afrique/ Europe (Mbembe, 2017, p. 18). L'intensification des migrations et l'établissement de nouvelles diasporas en divers lieux ont non seulement fait entré les populations africaines dans un âge de dispersion, de circulation et de mobilité vers des terres plus lointaines comme l'Amérique, mais elles ont aussi construit des pôles attractifs sur le continent noir même (Mazauric et Sow, 2013, p. 7). On parle alors de migrations transafricaines. Et l'écriture de Mabanckou consacre une large place à des images s'attelant à construire le personnage du migrant en tenant compte de toutes ces données que nous entendons observer dans la présente contribution.

L'intérêt de cette étude réside dans le fait qu'elle pourrait apporter quelques clés pour l'interprétation d'un imaginaire assurément performatif. Qui plus est, l'œuvre à laquelle elle s'applique se trouve être celle d'un acteur majeur de l'échiquier littéraire contemporain. La notoriété d'Alain Mabanckou est en effet difficile à démentir: il a été honoré par de nombreux prix prestigieux dont le Renaudot (2006), et sa nomination au Collège de France en 2016 (pour y tenir une Chaire de Création Artistique) constitue le couronnement d'un brillant parcours. Ceci ne saurait nous laisser indifférents.

\section{MÉTHODE}

L'étude que nous amorçons s'appuiera sur un corpus limité à cinq romans retenus en raison des occurrences significatives qu'on peut y déceler. Il s'agit notamment de Bleu blanc rouge (Mabanckou, 1998), Mémoires de porc-épic (Mabanckou, 2006), Les petits-fils nègres de Vercingétorix (Mabanckou, 2002), Verre cassé (Mabanckou, 2005) et Tais-toi et meurs (Mabanckou 2012).

Nous les analyserons selon une approche sociopoétique, conformément aux développements théoriques qu'en propose Bourdieu (2015). Il va sans dire que le champ d'exploration que composent les textes susmentionnés sera envisagé comme le lieu d'investissement des " habitus » du corps social de référence de l'auteur, mettant au jour son expérience de la vie au travers de l'écriture. Le décryptage dudit corpus apportera outre mesure un éclairage sur les raisons qui poussent le sujet migrant au départ, les structures figuratives par lesquelles Mabanckou le soumet au crible de la xénophobie et dépeint ses désillusions. Ce qui nous aidera à saisir, dans une ultime articulation, les sensibilités idéologiques de l'écrivain Congolais en tant qu'auteur diasporique. 


\section{RÉSULTATS ET DISCUSSION}

\section{L'hypostase du migrant: quête de l'exotisme ou exil forcé?}

Les contributions littéraires de l'auteur aux réflexions sur les migrations se réapproprient les discours public et médiatique inscrivant le phénomène dans une actualité brûlante, en mettant par ailleurs en scène des déplacements de personnages $\mathrm{d}^{\prime}$ un continent à un autre, $\mathrm{d}$ 'une région à une autre, d'un pays à un autre, des campagnes vers les villes ou vice-versa, afin d'élucider des situations plurielles occasionnant les mouvements migratoires. Il est par conséquent difficile de trouver un dénominateur commun aux multiples figures aventurières repérables dans ses romans.

Certains personnages d'Alain

Mabanckou quittent leurs milieux d'origine pour répondre à un déséquilibre homéostatique de leurs sociétés devenues à leurs yeux à la fois précaires et inhospitalières: «le Congo est par terre et [...] la viande de bœuf n'est plus à la portée de tout le monde » (Mabanckou, 2012, p. 56), dit par exemple Julien Makambo dans Taistoi et meurs pour légitimer son voyage en France. Pour sa part, Kibandi est obligé de fuir Mossaka, en compagnie de sa mère et du narrateur Ngoumba, afin d'échapper au lynchage des populations de cette localité qui ont mis fin aux jours de son père, un sorcier maléfique reconnu par ces villageois comme celui qui jetait son dévolu sur des innocents pour étancher sa soif de sang. Le narrateur en fait le témoignage en ces termes:

[Kibandi et sa mère] revinrent en toute discrétion au village après l'enterrement, préparèrent quelques affaires, décampèrent dès l'aube sans laisser de traces, ils suivirent l'horizon, échouèrent ici à Séképembé où je me trouvais déjà. Je les avais précédé dès que j'avais vu errer l'autre lui-même de mon jeune maître venu $\mathrm{m}^{\prime}$ annoncer le départ imminent de ce village du nord, je sus ainsi qu'il fallait foncer vers le sud, vers un village nommé Séképembé, voilà comment nous sommes devenus bien malgré nous des habitants de ce village d'accueil (Mabanckou, 2006, p. 108).

$\mathrm{D}^{\prime}$ autres personnages $\mathrm{du}$ romancier se projettent parfois vers un ailleurs qu'ils idéalisent fortement, qu'ils conçoivent comme un Eldorado. Ils se trouvent renforcés dans leurs idylles par des sujets dont l'expérience à l'étranger a fait « des modèles de réussite » au sein de leurs communautés d'origine, ou encore qui suscitent la fascination et le goût de l'exotisme chez leurs compères, parce que le discours qu'ils tiennent nourrit un enthousiasme collectif et contribue à la construction du «mythe de l'ailleurs » comme terre de l'emploi, de la richesse et du bonheur.

Dans Verre cassé en l'occurrence, L'Escargot entêté, propriétaire du bar Le crédit a voyagé, jouit d'une certaine célébrité auprès des siens, mais pour cela, il lui a fallu chercher sa réussite en dehors de son pays (ce qui était une "mode», souligne le narrateur), et parcourir l'Angola, le Gabon, le Tchad puis le Cameroun (Mabanckou, 2005, p. 92). De même, dans Les petits-fils nègres de Vercingétorix, dès que Vincent Komotié se présente sur la place publique dans son district d'Owetto, de nombreux convives l'entourent et le supplient de faire le récit de son aventure cubaine dont tout le village n'ignore pourtant plus les details. «Il était un des rares du district à avoir mis les pieds à Cuba » (Mabanckou, 2002, p. 144). Le récit de son voyage faisait rêver, donnait envie aux plus jeunes de découvrir Santiago de Cuba.

Dans le contexte de la représentation de l'Afrique subsaharienne, les romans de l'auteur, par ces quelques données jettent les bases d'une construction discursive faisant du migrant un individu en quête à la fois d'exotisme, d'un espace de mieux vivre, de ressources financières ou qui fuit la persécution. Mais celui-ci est aussi parfois obligé de partir pour des raisons familiales ou sociales. En effet, mariée à Kimbémbé, Hortense dans Les petits-fils nègres de Vercingétorix se doit de quitter Owetto pour s'installer à Batalébé, lieu où est affecté son mari (Mabanckou, 2002, p. 149) ; tandis que c'est pour poursuivre ses études qu'Amédée émigre de Séképembé vers la France dans Mémoires de porc-épic.

S'agissant justement de la France, il importe de souligner que les romans de Mabanckou sont truffés d'anecdotes et de 
témoignages qui ne sont que les résurgences d'un imaginaire social propre à l'Afrique francophone en générale et au Congo en particulier, appréhendant l'ancienne puissance coloniale comme le lieu de rêve par excellence. Comme le précise Kasende, l'histoire commune de la France et des pays francophones de l'Afrique subsaharienne à contribué à l'émergence de migrations conditionnées par le statut social et familial du migrant dans son pays d'origine, mais aussi par la disparité de la situation socioéconomique de ce dernier et celle de l'Hexagone (Kasende, s . d., p. 38).

En Afrique, des régimes politiques autoritaires, illégitimes et corrompus entretiennent une insécurité psychologique et une précarité économique endémique, souligne Kasende, qui plongent les populations dans le désespoir et les poussent au départ. Souvent clandestinement, en raison des nombreuses restrictions liées à l'attribution des visas. $\mathrm{Vu}$ d'Europe, ceci n'est pas toujours compris et Mabanckou le précise dans Le sanglot de l'homme noir quand il dit:

Les Européens comprendront-ils un jour ce qui se passe dans la tête d'un gamin d'Afrique lorsqu'il imagine ce continent du Nord, persuadé que c'est là-bas que son rêve deviendra réalité? J'ai vécu, moi aussi, dans ce songe à la fois agréable et trompeur (Mabanckou, 2012, p. 73).

Dans son premier roman Bleu blanc rouge, l'écrivain Congolais initie une réflexion sur la figure du migrant à travers son héros Massala-Massala, qui quitte son pays pour poursuivre son rêve de réussite en France. Et la plupart de ses textes suivants n'échappent pas à l'exercice de la représentation de personnages associant l'Europe (et en particulier la France) à une vie faite exclusivement d'agréments, de raffinement et de bonheur. Dans Tais-toi et meurs, la scénographie de la migration place Julien Makambo au centre d'un récit présentant le migrant comme un individu sans qualification, ni perspectives satisfaisantes dans son pays, donc obligé de transgresser les lois en faisant usage de faux papiers pour réaliser son rêve parisien: «fausse carte de séjour, fausse pièce d'identité [...] En recevant mes papiers par
DHL à Pointe-Noire, je n'en crus pas mes yeux [...], je les contemplais avec émerveillement » (Mabanckou, 2012, p. 50).

Le bilan transversal des raisons qui poussent le migrant au départ révèle une fibre dialectique faisant de l'aventure migratoire soit une initiative motivée par la poursuite d'un idéal, soit une nécessité absolue au regard de "l'hostilité ou de la capacité d'agression » (Mengue-Nguéma, 2009, p. 35) du lieu d'établissement d'origine des personnages. Retenons pour l'essentiel l'incapacité des personnages de Mabanckou à se sédentariser dans leur milieu, parce que compromettant ou désolant. Sa forte négativité les conduit à manifester un besoin de mobilité qu'il convient de définir comme hypostase du sujet migrant, lequel migrant, une fois arrivé en terre d'accueil, doit souvent faire l'amère expérience de la xénophobie.

\section{Le migrant soumis au crible de la xénophobie}

Les romans $\mathrm{du}$ corpus à l'étude révèlent un traitement spécifique des stéréotypes communautaires liés aux conceptions traditionnelles de groupes ethniques et raciaux. De ce fait, les relations entre migrants et natifs du lieu d'accueil de ceux-ci sont souvent viciées par une métastase de la violence transversale, qu'elle soit verbale ou physique, révélant de profondes inimitiés. Nous sommes d'avis comme Bah (2005) que

L'histoire de l'humanité nous enseigne que les rapports ou les rencontres interindividuelles, inter-communautaires et surtout inter-nationales ne sont pas, d'emblée, le champ de la communion, de la fraternité, de la solidarité et de l'amour. Elles donnent lieu au conflit: le conflit des cultures. Au cours de ces rencontres, le déséquilibre dans les capacités d'action [...] procure aux uns des avantages dont ils profitent au détriment des autres (p. 159).

Dans Les petits-fils nègres de Vercingétorix, Hortense se sent étrangère à Batalébé. Elle est ressortissante du nord du Viétongo mais s'est installée dans ce village du sud, duquel est natif son mari Kimbémbé sans parvenir à se faire accepter, excepté par 
Christiane (sa meilleure amie en ce lieu), dont l'époux est de la même région que l'héroïne. Le mariage donne souvent certains droits à la femme, notamment celui de pouvoir acquérir la nationalité de son mari (ici c'est la région), mais Hortense demeure étrangère dans le village de Kimbémbé. Elle n'extériorise cependant pas ses sentiments et préfère les consigner dans ses cahiers. L'écriture devient en quelque sorte pour elle une soupape de sécurité pour trouver un équilibre dans un environnement qu'elle perçoit comme austère.

Son esprit est d'autant plus mortifié que son mari, autrefois si aimant, manifeste lui aussi désormais à son égard une haine xénophobe après avoir été endoctriné. Quand le président Labou Kabouya, originaire du sud du Viétongo est destitué par le général Edou, issu du nord comme Hortense, Kimbémbé se radicalise. Ce bouleversement politique a des répercussions sur le foyer conjugal d'Hortense: elle se sent désormais en insécurité avec un époux devenu intolérant. L'héroïne dit à ce sujet: « Le président Labou Kabouya avait-il autant souffert que Kimbémbé? J'en doute encore. En tout cas le moindre mot que je prononçais l'enflammait. Je n'avais rien à dire à ce sujet au risque d'être traitée d'espèce de nordiste " (Mabanckou, 2002, p. 192).

L'auteur met ainsi en surface le problème de l'exclusion dont est victime le migrant. Cette exclusion est ici liée à la célébration par certaines personnes d'un culte immodéré pour le groupe ethnique auquel elles appartiennent. Les habitants de Batalébé entretiennent l'idée d'une factice supériorité de leur ethnie sur celle d'Hortense Iloki: « Tu leur pose un problème et tu dois le savoir... » (Mabanckou, 2002, p. 44), lui fait remarquer Christiane. Bien entendu cette intolérance xénophobe inhérente à la revendication d'une identité ethnique ne peut garantir la quiétude du sujet migrant, ce qui poussera Hortense à s'enfuir à Louboulou dans l'espoir de rejoindre Pointe-Rouge.

Le migrant est donc chez notre auteur exposé à une hostilité systémique et irrationnelle en raison de son groupe d'appartenance. Enraciné dans les mœurs à la manière d'un arbre dans le sol, le sentiment tribal se découvre dans le comportement des personnages de Mabanckou. Dans le contexte d'une crise sociopolitique, il engendre le conflit interethnique qui structure l'intrigue du roman Les petits-fils nègres de Vercingétorix. Gaston Okemba, « nordiste » qui a immigré à Batalébé comme Hortense, paiera de sa vie cette conception symbolique de «l'étrangerennemi», envisagé comme figure « négative » par les habitants de Batalébé et les miliciens du chef de guerre Vercingétorix. Ces derniers ne manqueront pas de lui signifier que dans ce village il n'est pas "chez lui ", avant de l'enlever et de l'éliminer (Mabanckou, 2002, p. 51).

Et le constat de la violence identitaire suscitée par le repli communautaire est élargi à la sphère raciale, quand l'auteur dépeint le migrant africain en Europe. C'est pour lui une manière de recentrer l'histoire du face à face entre le Noir et le Blanc. Dans Tais-toi et meurs le romancier confère au migrant Africain en France une vraie conscience de soi, mais dans le même temps il le laisse aussi être vu au travers des «Blancs». Ceci débouche sur une réévaluation du paradigme des théories racialistes soumettant l'immigré à l'épreuve de la stigmatisation. Ces théories qui, face au chômage, à la crise économique et l'insécurité grandissante en France, proclament que le « recul » de cette nation est le fait de l'autre, cet autre (de race noire notamment) qu'on ne voit dans les journaux télévisés que lorsqu'il a transgressé la norme sociale, ou dans des reportages qui le montrent vivant dans des immeubles insalubres «où il a importé ses mœurs primitives» (Mabanckou, 2012, p. 90).

Appréhendé comme suspect suite à la mort d'une jeune fille par défénestration, Julien est traité de « Fils de pute! Sale négro » (Mabanckou, 2012, p. 26). La couleur de la peau est ici à l'évidence un motif de dénigrement et de rejet, d'autant que dans la bouche d'un autre personnage sortiront des paroles stigmatisantes à l'encontre des Noirs, s'inscrivant dans le cadre d'un discours performatif et protectionniste: «- On les 
accepte dans ce pays et voilà ce qui nous arrive ! Où est donc passée la justice, hein? Et on s'étonne que les gens votent massivement pour l'extrême droite ces derniers temps!» (Mabanckou, 2012, p. 92). Ces propos qui attribuent aux migrants négroïdes en provenance de l'Afrique la responsabilité de la recrudescence du banditisme, régis par la rhétorique des appartenances, révèlent que de plus en plus de citoyens Français «blancs» (si l'on peut ainsi s'exprimer) pensent qu'il faudrait défendre la France contre les migrants, envahissants et déstabilisateurs, ignorant la multiplication des interférences entre les peuples, et pardelà, la complexité de cette ère nouvelle qui nous lie les uns aux autres, loin des considérations géographiques et raciales.

La figure du migrant, mise à l'épreuve de la xénophobie dans le corpus, pose la question du rapport à l'autre et des phénomènes ambivalents que sont l'ethnocentrisme, le racisme et le patriotisme exacerbé. Pour une époque telle que la nôtre, assise sur des certitudes scientifiques perçues comme le signe du "progrès" de nos sociétés, malgré les doutes dans lesquels nous plongent de nombreux fléaux, l'auteur montre combien nous serions avisés de revenir sur nos pas et de retourner vers un humanisme universel (Mbem, 2007, p. 7) qui transcende les différences, pour s'inscrire dans la réalité immédiate. Et cette réalité fait avant tout de ces mêmes sociétés des espaces d'expression de la diversité.

\section{Le migrant et l'effondrement du mythe de l'Occident}

La représentation du sujet africain immigré en Occident correspond aussi chez Mabanckou à ce qu'on pourrait assimiler à une structure isotopique qui sert de soubassement à la littérature migratoire. Cette structure met en évidence un décalage entre les espoirs de réussite et de promotion sociale qui motivent le migrant et la dure réalité de ce continent qu'il fini par découvrir. Il en résulte un sentiment de désillusion en ce sens que l'Europe qu'il découvre n'est pas conforme à l'imagerie qu'il s'en faisait depuis l'Afrique: c'est l'effondrement du mythe de l'Occident.
En empruntant le chemin de l'Europe, le migrant, jeune la plupart du temps, a souvent l'impression qu'il débouchera sur une clairière où sa misère prendra fin comme on le voit dans Bleu blanc rouge avec MassalaMassala. L'Eldorado tant espéré n'offre cependant pas toujours autant d'opportunités qu'il ne le pense. L'embauche n'y est pas garantie, se loger et se nourrir décemment sont loin d'être à la portée de tout le monde. C'est l'autre visage de l'Europe qu'il découvre. Une Europe avec ses chômeurs, ses sans domicile fixe, ses résidents précaires des HLM et ses sans papiers obligés de se cacher pour échapper aux contrôles policiers.

Dans Tais-toi et meurs, le lieu de résidence de Julien et ses amis est symptomatique de l'inconsistance de leur condition en tant qu'immigrés: un studio exigu pour six personnes sans emploi stable (Mabanckou, 2012, p. 55), obligées chacune de «se débrouiller» pour contribuer au paiement du loyer et assurer les besoins primaires au quotidien. Julien Makambo qui voulait à tout prix tenter sa chance en France, réalise que séjourner à Paris a un prix qui ne s'évalue pas toujours en termes d'argent, qui porte parfois un coup à la dignité humaine. Le retour au pays étant difficile, voire impossible, il faut dès lors revoir ses plans, trouver d'autres voies de recours pour survivre.

Son compatriote Willy, mécanicien au Congo, s'était lui rendu en Fiance dans l'espoir de travailler chez un grand constructeur automobile comme Renault ou Citroën. Mais l'intégration de technologies pointues dans la conception des véhicules de ces concessionnaires lui fait prendre conscience qu'il ne suffit plus, comme il le faisait dans son pays natal, de bidouiller un boulon ou une vis pour réparer une automobile. Il faut désormais avoir un certain niveau de qualification et une réelle expertise. Or Willy, sans diplôme en la matière, a appris le métier sur le tas dans le garage de son oncle maternel. Il est donc obligé de revoir ses prétentions à la baisse en travaillant avec d'autres immigrés qui réparent à la sauvette des voitures à Montreuil (Mabanckou, 2012, p. 45-46). 
De même, Désiré dans ce même roman voit ses rêves de succès dans une carrière musicale s'effriter. Croyant que la France lui offrirait l'occasion de devenir célèbre, il en est réduit à jouer de la guitare dans le métro parisien pour quelques pièces de monnaie. L'idée paradisiaque que ces personnages avaient au départ de la France se transforme en cauchemar et l'étau de l'étranger se resserre autour d'eux. Clandestins, les voilà pris malgré eux dans un engrainage, un cercle vicieux que décrit si bien Kasende (s. d.): pour trouver un travail convenable il faut avoir des papiers et pour avoir des papiers il faut un travail. Les turpitudes de ces personnages liées à l'immigration contredisent l'idée d'un paradis européen.

La figure du migrant apparait dans le corpus comme un catalyseur qui, au sein du texte, engage une réflexion sur des processus qui mettent en relief l'illusion et le paradoxe de l'aventure migratoire en Occident. Ce qui permet au lecteur de procéder à une réévaluation du mythe qui l'entoure. "Pris entre le marteau du rêve occidental et l'enclume de la triste réalité » (Diandue, 2005, p. 21), le personnage du migrant Africain en Europe se met au service d'un discours visant à interpeller l'opinion publique sur des réalités liées à la condition humaine. Les points de fixation qui érigent le migrant en figure majeure des romans d'Alain Mabanckou sont redevables à la fois à notre époque et à un projet esthétique rigoureux, qui se propose $\mathrm{d}^{\prime}$ associer toute exégèse et tout commentaire sur la question des migrations à des considérations éthiques qu'il assume en sa qualité d'écrivain de la diaspora.

\section{Éthique normative et engagement diasporique de Mabanckou}

Dans ses travaux les plus récents sur la littérature africaine, Toivanen (2011) souligne qu'en ce XXIème siècle cette littérature porte le sceau des affinités transnationales. La théorie postcoloniale, hégémonique, a de son point de vue consolidé le paradigme postcolonial en mettant un accent particulier sur les métaphores délocalisées et nous adhérons pleinement à son postulat. Ce qu'elle fait remarquer au sujet du roman
Celles qui attendent de Diome (2010) nous paraît convenir aussi, en certains points, à l'œuvre d'Alain Mabanckou, d'autant que les itinéraires des deux auteurs présentent de considérables similitudes.

Comme la romancière Sénégalaise, Alain Mabanckou donne à l'engagement littéraire une impulsion en adéquation avec les enjeux du monde contemporain. Suite à la contestation du système colonial qui a débouché sur les indépendances puis le désenchantement qui s'en est suivi, le nouvel ordre mondial impose une réorientation de l'engagement des auteurs de la diaspora. Pour mémoire, la littérature africaine écrite est la résultante de la rencontre de deux mondes. Le contact de l'Afrique avec l'Occident a conditionné une relation à l'imaginaire dans la représentation de l'autre. Le tout sur fond de colonisation, avec sa cohorte de dispositifs discriminatoires et répressifs: indigénat, travail forcé, etc.

Dans les années 50 les langues se délient, les productions littéraires se multiplient et «s'affirment de plus en plus dans la contestation ", comme le note Kesteloot (2001, p. 204-205). Et quand l'euphorie des indépendances acquises dans la boue et dans le sang se dissipe pour laisser la place à la désillusion et à l'afropessimisme, la technique d'écriture devient plus élaborée et les personnages acquièrent une certaine «épaisseur » qui donne de la matière à la critique pour des analyses plus riches. Depuis les textes de réprobation et de désaliénation dont des écrivains comme Oyono (1956),Tansi (1979), Sembene (1973) sont des icônes, l'espace littéraire africain est devenu un champ dynamique de l'expression de l'engagement.

Or, dans le contexte actuel qui met en scène les auteurs de la Migritude, l'engagement s'exprime davantage sur le plan éthique que sur le plan strictement politique. Ainsi, au lieu de faire la promotion d'un agenda politique, les personnages de l'univers romanesque de l'écrivain Congolais sont au centre de récits de nature à soulever une réflexion sur la reconnaissance de la pluralité des voix, des identités, et l'acceptation des désaccords que cela pourrait occasionner au nom de la tolérance. 
Les images en rapport avec la question des migrations chez Alain Mabanckou répondent à un traitement critique des disparités socioéconomiques qui contentent le monde occidental mais continuent à faire des exclus parmi les Africains, voire les populations du Sud plus largement. Or il est légitime pour tout individu d'aspirer à une vie meilleure. Dans un article signé conjointement avec Wabéri, Mabanckou indique que le continent noir « est sans doute un des laboratoires par excellence pour quiconque veut observer l'évolution actuelle de la mondialisation [et des phénomènes migratoires] » (Mabanckou et Waberi, 2017, p. 239). Il fait remarquer, sans devoir remonter au déluge, que l'émigration $\mathrm{n}^{\prime} \mathrm{y}$ est pas un problème nouveau.

Il $\mathrm{y}$ a en Afrique d'importants déplacements transnationaux et transcontinentaux des populations, mais il faut se rappeler qu'à l'échelle des nations elles-mêmes, les migrations internes qui se manifestent par le truchement de l'exode rural ont pendant longtemps dépeuplé les campagnes et engorgé les centres urbains, mieux équipés et offrant davantage de perspectives. Pour Mabanckou la situation actuelle est similaire, sauf qu'elle se pose dans des proportions plus grandes et implique des espaces plus élargis. Si l'attractivité des villes a contribué au dépeuplement des villages, par analogie fonctionnelle le développement de l'Occident qui jouit par ailleurs pleinement des atouts offerts par la mondialisation ne peut que susciter des velléités de départ chez nombre de populations du Sud, désœuvré et déshérité. Même si cet « ailleurs » tel qu'elles le conçoivent ne constitue parfois qu'un mirage.

Dans Tais-toi et meurs Julien Makambo est un congolais qui se rend en France clandestinement et qui désire se faire une place dans un monde qui finalement « l'écrase ». L'imprimeur dans Verre cassé est ce qu'on appelle au Congo «un parisien refoulé », parce que expulsé de France injustement. Pourtant les discours officiels sur les migrations sont des plus polis: il faut plus $\mathrm{d}^{\prime}$ "aide au développement ", de la cooperation «gagnant/ gagnant», faire du monde un village planétaire en brisant les frontières et $\mathrm{j}$ 'en passe...

Mais, il s'agit de briser les frontières culturelles précise-t-on, tandis que les frontières géographiques, elles, sont renforcées pour faire de l'Occident richissime une forteresse, comme en témoigne ce qu'on appelle aujourd'hui «le drame de la méditerranée ». Alain Mabackou jouit d'un succès qui va au-delà du continent africain et il est traduit dans près d'une dizaine de langues. Ceci lui a permis de se positionner dans le canon littéraire mondial. Le lecteur de son œuvre appartient de ce fait tant à la sphère locale africaine que globale ou mondiale. En vertu de cela, les aventures de ses personnages, telles qu'elles sont bâties, interpellent aussi bien l'Occident, la diaspora, que ceux dont les vies sont affectées sur le continent noir. En conséquence sa position d'auteur est profondément «marquée par une complexité qu'on ne peut pas comprendre en seuls termes d'opposition mutuellement exclusives » (Toivanen, 2011., p. 74).

Ayant acquis la citoyenneté française et passant une grande partie de son temps aux Etats Unis où il enseigne les littératures africaine, antillaise et française, Mabanckou a aussi beaucoup voyagé en Afrique. La « multi culturalité » qu'il a développée et qu'il met au jour dans Le monde est mon langage (2016) le consolide dans une approche créatrice donnant lieu à des romans dont les voix narratives émanent de personnages qui ne condamnent pas nécessairement telle partie au détriment d'une autre, qui ne revendiquent pas forcément une idéologie pour en réprimander une autre. Trouver le parfait équilibre dans la complexité de ce problème en tant qu'auteur diasporique, c'est l'ambition de sa poétique et elle est portée par des protagonistes continuellement en déplacement.

Au-delà des paramètres économiques auxquels elles ne sauraient être réduites, les migrations nous paraissent être au service de transformations multiformes pour le monde, au point de revêtir la puissance anonyme du destin que chaque individu souhaiterait se construire. Au lieu d'accueillir des discours 
sermonneurs ou conservateurs en la matière, les romans de Mabanckou, par les personnages auxquels ils procurent des espaces de mobilité et d'expression, révèlent une critique des idées qui a vocation à susciter un regard distancié et nuancé, dépouillé des clichés et préjugés en tous genres, pour s'ouvrir à un humanisme universel, lui-même mouvant et fluctuant. Ainsi signe-t-il son engagement diasporique par un propos modulé, en réponse à l'exclusion et à la passion que génèrent aujourd'hui les questions migratoires:

J'ai choisi depuis longtemps de ne pas m'enfermer, de ne pas considérer les choses de manière figée, mais de prêter plutôt l'oreille à la rumeur du monde. Je ne suis pas devenu écrivain parce que j'ai quitté mon pays natal. En revanche, j'ai posé un autre regard sur celui-ci une fois que je m'en suis éloigné [...]. Le déplacement a contribué à renforcer en moi cette inquiétude qui fonde à mes yeux toute démarche de création. On écrit parce que quelque chose ne tourne pas rond, parce qu'on voudrait remuer les montagnes ou parce qu'on voudrait introduire un éléphant dans le chas d'une aiguille. L'écriture devient alors à la fois un enracinement, un appel dans la nuit et une oreille tendue vers l'horizon (Mabanckou, 2016, p. 11).

\section{CONCLUSION}

L'écriture d'Alain Mabanckou est particulièrement marquée par une réflexion quasi obsédante sur les migrations. Il est vrai que « dans les productions littéraires africaines, le déplacement, la migration et le voyage jouent depuis toujours un rôle éminent » (Mazauric et Sow, 2013, p. 10), mais les situations qui lui servent à énoncer la mobilité et à construire la figure du migrant sont aussi variées que complexes. En portant une attention plus grande à cela, on se rend compte que l'écriture de la migrance chez Mabanckou répond à des logiques paradoxalement archaïques et contemporaines. Cette ambivalence est justement redevable à la complexité de cette problématique qui pose aux espaces d'intersection des peuples le défi de la construction de communautés paisibles. Le romancier Congolais dénonce en effet l'hostilité dont les migrants sont souvent victimes. De son point de vue elle repose sur un savoir apparent fait de préjugés qui occasionnent toutes sortes de discriminations.

Pour autant, l'auteur ne passe pas sous silence les impasses existentielles sur lesquelles peut déboucher l'aventure migratoire. Les épreuves qui marquent le parcours de ses personnages placent ainsi ces derniers en situations d'apprentissage, que leur choix de partir soit délibéré ou imposé par l'implosion (guerre) ou la négativité (précarité) de leurs lieux d'établissement d'origine. Le but est vraisemblablement celui de créer des conditions qui pourraient permettre au lecteur de poser un regard plus nuancé sur ce phénomène et de se départir d'une rhétorique du rejet de l'Autre simplement du fait de sa différence.

Les textes du corpus étudié mettent par conséquent en exergue un positionnement éthique auctorial postulant la nécessité de repenser et de redéfinir nos rapports à autrui, les différences ethniques et raciales ne pouvant justifier le cynisme et la violence. La tolérance reste donc pour Mabanckou une des voies pour l'avènement d'un monde en harmonie avec lui-même.

\section{REMERCIEMENTS}

Je tiens à remercier l'équipe de la revue FRANCISOLA pour la fructueuse collaboration qui a debouche sur cette pubication. Que trouvent également ici l'expression de ma sincère gratitude le Professeur Mosé Chimoun (mon directeur de thèse), Delphe Kifouani, Maître de Conférences à l'Université Gaston Berger, pour leurs précieux conseils. Je n'oublie pas Joseph Martial Bassintsa, car le soutien inconditionnel $\mathrm{d}^{\prime}$ un père quand on s'engage dams une enterprise aussi périlleuse que la recherché universitaire est d'une préciosité inestimable. Enfin, merci à mon épouse Natalie qui s'est prêtée volontier à la relecture de cette contribution pour en corriger les coquilles, ainsi qu'à Prosper Faye pour avoir assuré la traduction du résumé de cet article en anglais. 


\section{RÉFÉRENCES}

Albert, C. (2005). L'immigration dans le roman francophone contemporain. Paris: Karthala.

Bah, H. (2005). Mondialisation de la culture et figures de l'altérité. Ethiopiques, (74), 159-173.

Bourdieu, P. (2015). Les règles de l'art: genèse et structure $d u$ champ littéraire. Paris: Points.

Diandue, B. K. P. (2005). Le ventre de l'atlantique, métaphore aquatique d'un mirage: idéal brisé de l'ailleur?. Ethiopiques. (74), 15-27.

Diome, F. (2010). Celles qui attendent. Paris: Art Editions

Kasende, J. C. (s.d.). (E)Migration et imaginaire social africain dans Le ventre de l'Atlantique de Fatou Diome: Construction discursive en référence au mythe de L'Odyssée. Etudes Francophones. 26(1-2), 38-55. Repéré à https://languages.louisiana.edu/sites/la nguages/files/kasende26EF.

Kesteloot, L. (2001). Histoire de la littérature négro-africaine. Paris: Karthala.

Mabanckou, A. (1998). Bleu blanc rouge. Paris: Présence Africaine.

Mabanckou, A. (2002). Les petits-fils nègres de Vercingétorix. Paris: Le Serpent à Plumes.

Mabanckou, A. (2005). Verre Cassé. Paris: Seuil.

Mabanckou, A. (2006). Mémoires de porc-épic. Paris: Seuil.

Mabanckou, A. (2012). Tais-toi et meurs. Paris: La Broche.

Mabanckou, A. (2012). Le sanglot de l'homme noir. Paris: Fayard.
Mabanckou, A. (2016). Le monde est mon langage. Paris: Grasset.

Mabanckou, A. et Waberi, A. (2017). Dictionnaire amoureux du continent africain: deux entrées. Dans A. Mbembe et F. Sarr (dir), Écrire l'Afrique-Monde (p.234-241). Dakar: Philippe Rey / Jimsaan.

Malonga, A. N. (2007). Roman congolais Tendances thématiques et esthétiques. Paris: L'Harmattan.

Mazauric, C. et Sow, A. (2013). Littérature et migrations transafricaines. Études Littéraires Africaines. (36), 7-16.

Mbem, A. J. (2007). La quête de l'universel dans la littérature africain De Léopold Sédar Senghor à Ben Okri. Paris: L'Harmattan.

Mbembe, A. (2017). L'Afrique qui vient. Dans A. Mabanckou (dir.), Penser et écrire l'Afrique aujourd'hui (p.17-31). Paris: Seuil.

Mengue-Ngéma, R. M. (2009). La représentation des conflits chez Ahmadou Kourouma et Alain Mabanckou (19982004) (Thèse de Doctorat, Université de Chergy-Pontoise). Repéré à http://biblioweb.ucergy.fr/theses/09CERG0413.pdf

Oyono, F. (1956). Une vie de boy. Paris: Julliard.

Sembene, O. (1973). Le docker noir. Paris: Présence Africaine.

Tansi, S.L. (1979). La vie et demie. Paris: Seuil

Toivanen, A. L. (2011). Retour au local: Celles qui attendent et l'engagement diasporique de Fatou Diome. Repéré à https://www.revuerelief.org/articles/10.18352/relief.658/ 